\title{
CERN to test revolutionary mini-accelerator
}

\section{Plasma wakefield machines aim to reach high energies without huge gains in size.}

\section{BY ELIZABETH GIBNEY}

$\mathrm{T}$ The home of the Large Hadron Collider (LHC), the world's largest particle accelerator, is getting a new machine - and this time, the whole point is to keep it small.

On 18 September, the council that governs CERN, Europe's premier particle-physics laboratory, near Geneva, Switzerland, approved a boost in funding for a planned experiment called the Advanced Wakefield Experiment, or AWAKE. Due to switch on next year, AWAKE will accelerate particles by 'surfing' them on waves of electric charge created in a plasma, or ionized gas. It is a method that could allow future accelerators to probe matter and the forces of nature at ever-higher energies, without the usual accompanying increase in the instruments' size and therefore cost.

Although plans are afoot to build bigger machines once the LHC reaches the end of its life in the 2030s (see go.nature.com/a9sm2m), many fear that accelerator size is nearing its limit and that such proposals may simply prove too expensive to implement.

"When you look at cost estimates for these machines and the scale of machines, you understand that maybe a new breakthrough regime is needed," says Nick Walker, an accelerator physicist at DESY, Germany's high-energyphysics laboratory in Hamburg.

Conventional colliders, such as the 27-kilometre-long LHC, use electric fields to move charged particles through a tunnel; the fields switch from positive to negative at a frequency that means the particles are constantly nudged forward, gaining energy with each push. But such colliders use metal-walled cavities that spark if the electric field is too strong. As a result, the only way to further increase the particles' speed, and therefore energy, is to lengthen the tunnel.

Plasma wakefield accelerators, which were first proposed in the 1970s, are designed to break this cycle, says physicist Allen Caldwell at the Max Planck Institute for Physics in Munich, Germany, who will lead the AWAKE experiment. They send a pulse of charged particles or laser light through a plasma, which sets electrons and positively charged ions oscillating in its wake. The resulting regions of alternating negative and positive charge form waves that accelerate further charged particles. Injected

\section{WAKEFIELD ACCELERATION}

The AWAKE experiment at CERN will test whether pulses of protons can be used to turn a plasma-filled machine into a particle accelerator.

\section{MAKING WAVES}

A pulse of protons injected into an ionized gas, or plasma, sets electrons bobbing in its wake, creating regions that constantly cycle between being positively and negatively charged.
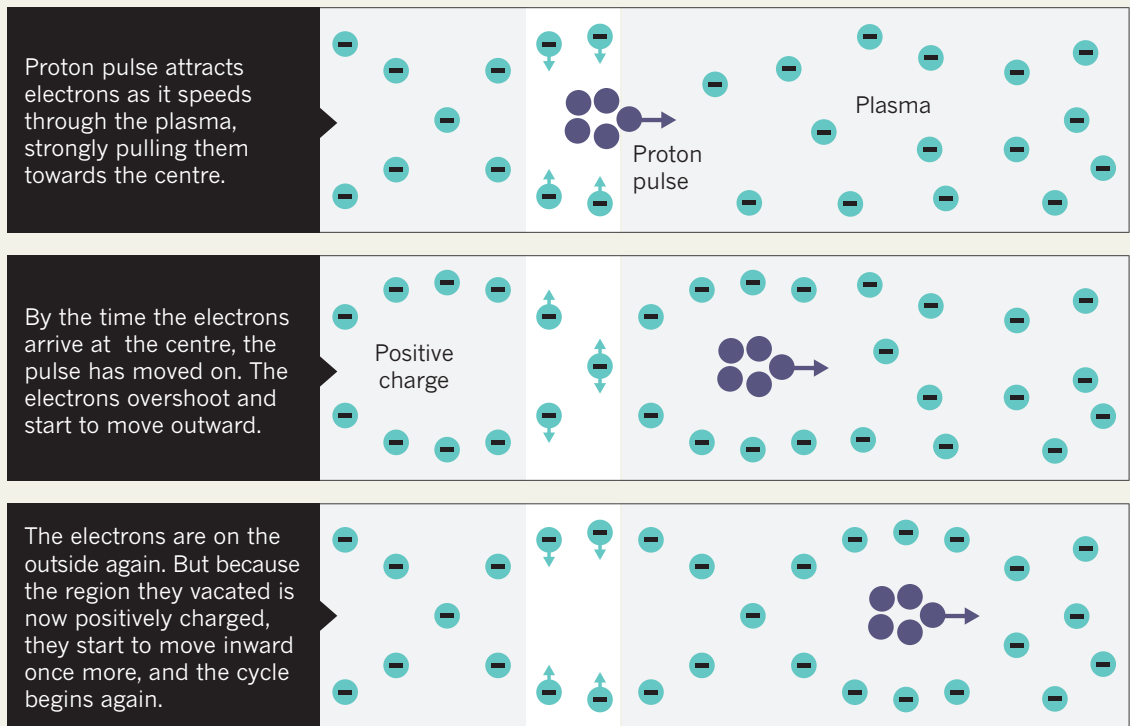

\section{SURF'S UP}

The cycling from positive to negative charge creates a wave of electron density that can be used to accelerate injected electrons.

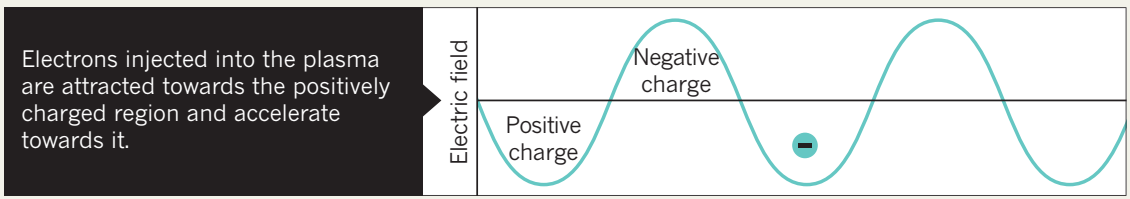

But that region quickly becomes negatively charged, forcing the electrons to accelerate again to stay in the positively charged region - rathe like a surfer who is constantly on the downward slope of a moving wave.

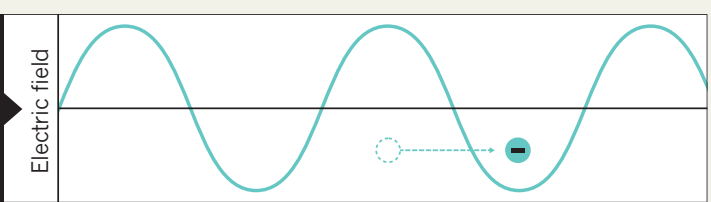

at just the right time, these particles effectively surf the waves (see 'Wakefield acceleration'). Crucially, as the electric fields are much stronger than those in a conventional collider, the acceleration can be as much as 1,000 times greater over the same distance.

Such accelerators exist in prototype at several facilities around the world, but AWAKE will be the first time that CERN has experimented with the technology. "CERN is the world's high-energy physics lab right now, and the fact that it has decided this is an important field to get involved in is a bit of validation for this community," says Mark Hogan, an accelerator physicist at the SLAC National Accelerator Laboratory in Menlo Park, California. 
Different groups have different ways of setting the plasma oscillating: Hogan's team at SLAC uses pulses of electrons, for example. AWAKE will be the first to use pulses of protons, which have some big advantages.

Because protons have greater mass than electrons, each proton pulse penetrates further into the plasma, setting up a longer series of charged regions, which in turn provides greater acceleration per pulse. A proton machine is also compatible with the LHC, which accelerates and collides protons.

For now, AWAKE will use the proton bunches that feed the LHC to test whether protons can generate the electric fields necessary to accelerate particles in plasma.

The latest investment from CERN worth 2.6 million Swiss francs (US\$2.7 million), from the total of 21.4 million Swiss francs so far committed to the experiment - is intended to allow AWAKE to test the concept before the end of 2018, when CERN is scheduled to shut down its accelerators for an upgrade. Success will depend on whether these proton bunches,

"The fact that CERNhas

decided this is an important field to get involved in is a bit of validation for this community." which are long relative to what is needed to create plasma waves, can be efficiently chopped up into short pulses.

Eventually, it might be possible to inject the much-higher-energy protons that have been accelerated by the LHC into a plasma wakefield machine for further acceleration. Hogan estimates that a machine just a few kilometres long could produce electrons with 6 times the energy of those that would be produced by the next planned conventional accelerator, the 31-kilometre-long International Linear Collider.

Despite such promise, plasma accelerators are decades from practical use because, to do better than existing accelerators, they must also match them in efficiency - supplying focused, accelerated particles at high rates as well as high energies, says Walker. Still, he adds, "right now, this is the only thing I see that might work".

The technology might also be useful elsewhere. Wakefield-accelerated electrons could drive X-ray free-electron lasers, which probe matter using powerful bursts of light that are short enough to capture the motions of molecules. These are currently kilometres long - but using wakefield technology might allow them to fit into labs or hospital basements. "I think this is more realistic as a potential application," says Walker, "and I would say a mandatory first step before the plunge into trying to achieve high-energy-physics experiments." -
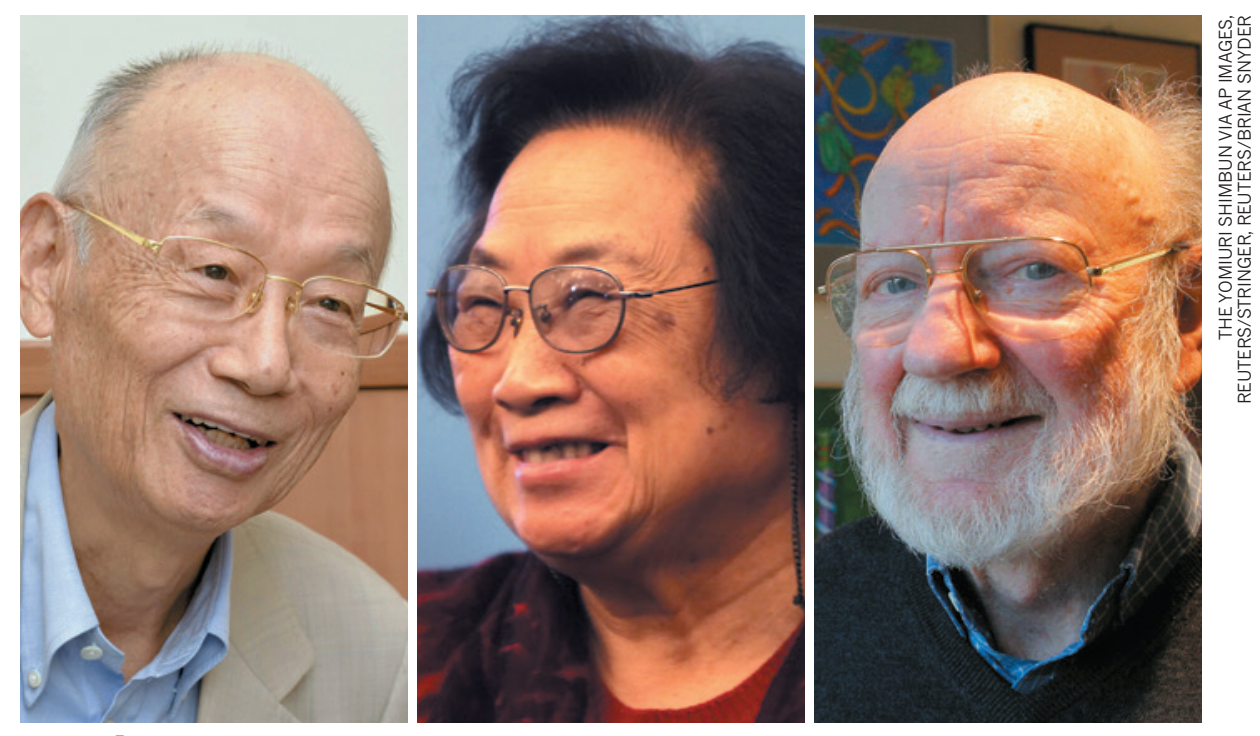

Satoshi Ōmura, Youyou Tu and William C. Campbell share the Nobel Prize in Physiology or Medicine.

MEDICINE

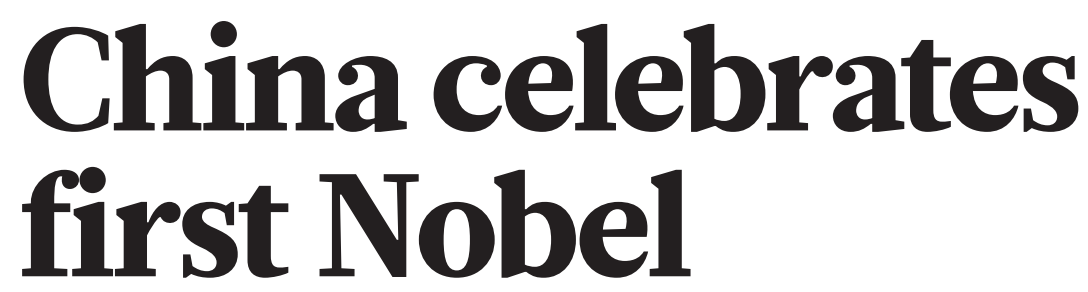

\section{Pharmacologist shares prize for work on parasitic infections.}

\section{BY EWEN CALLAWAY \& DAVID CYRANOSKI}

$\mathrm{F}$ or the first time, a researcher based in China has won the ultimate status symbol in science - a Nobel prize.

Pharmacologist Youyou Tu, who led a Beijing team that discovered the key malaria drug artemisinin in the late 1960s and 1970s, was awarded the 2015 Nobel Prize in Physiology or Medicine on 5 October. Two microbiologists, William C. Campbell at Drew University in Madison, New Jersey, and Satoshi Ōmura at Kitasato University in Japan, shared the award for their development - also in the 1970s — of therapies against parasitic roundworms.

"This certainly is fantastic news for China. We expect more to come in the future," says Wei Yang, president of the nation's main research-funding agency, the National Natural Science Foundation of China. Lan Xue, an innovation-studies specialist at Tsinghua University in Beijing, says that he was inundated with messages about the prize. "People will be celebrating, but I hope they also take a sober look, because there are lots of things to learn from this award," he says.

Young scientists in China today are told to go overseas to do good research and to churn out publications in internationally recognized journals, Xue notes. Yet Tu has never worked outside China, and has not racked up major publications. "Tu doesn't fit into any of the trends today, and yet she gets the Nobel because of the originality of her work. It couldn't have been a better choice in terms of the lessons it offers Chinese scientists," Xue says.

\section{MALARIA BREAKTHROUGH}

Tu's prizewinning research, at the China Academy of Chinese Medical Sciences in Beijing, originated from a government push in 1967 to discover new therapies for malaria. At the time, the main treatments - chloroquine and the older quinine - were proving increasingly ineffective. Tu and her team screened more than 2,000 Chinese herbal remedies to search for drugs with antimalarial activity. An extract from the wormwood plant Artemisia annua proved especially effective, and by 1972 , the researchers had isolated chemically pure artemisinin.

"I'm very happy about this. She totally deserves it," says Yi Rao, a neuroscientist at Peking University in Beijing who has researched the discovery of artemisinin. But there has been some controversy over credit for the discovery, Rao points out, so Tu has never won a major award in China. She has not been elected to either of China's major academies neither the Chinese Academy of Sciences nor the Chinese Academy of Engineering.

"Though other people were involved, Tu 